ANNALES

POLONICI MATHEMATICI

LXXVII.1 (2001)

\title{
Opial inequalities on time scales
}

\author{
by MARTin Bohner (Rolla, MO) and \\ BílLÛR KaYMaKÇALAN (Ankara)
}

\begin{abstract}
We present a version of Opial's inequality for time scales and point out some of its applications to so-called dynamic equations. Such dynamic equations contain both differential equations and difference equations as special cases. Various extensions of our inequality are offered as well.
\end{abstract}

1. Introduction. Opial inequalities and many of their generalizations have various applications in the theories of differential and difference equations. This is very nicely illustrated in the book [2] "Opial Inequalities with Applications in Differential and Difference Equations" by Agarwal and Pang, which currently is the only book devoted solely to continuous and discrete versions of Opial inequalities. In this paper we present several Opial inequalities that are valid on so-called time scales. The calculus of time scales has been introduced by Hilger [8] in order to unify discrete and continuous analysis. Hence our results cover both the continuous (originally by Opial [12]) and discrete (originally by Lasota [11]) Opial inequalities as given in [2].

For convenience we now recall the following easiest versions of Opial's inequality.

Theorem 1.1 (Continuous Opial Inequality, [2, Theorem 1.4.1]). For absolutely continuous $x:[0, h] \rightarrow \mathbb{R}$ with $x(0)=0$ we have

$$
\int_{0}^{h}|x \dot{x}|(t) \mathrm{d} t \leq \frac{h}{2} \int_{0}^{h}|\dot{x}|^{2}(t) \mathrm{d} t,
$$

with equality when $x(t)=$ ct.

2000 Mathematics Subject Classification: 34A40, 39A13, 26 D15.

Key words and phrases: time scales, Opial inequality, Lasota inequality, dynamic equations.

Research of the second author done while visiting UMR supported by a NATO-B2 Scholarship of TÜBITAK (Turkey). 
Theorem 1.2 (Discrete Opial Inequality, [2, Theorem 5.2.2]). For a sequence $\left\{x_{i}\right\}_{0 \leq i \leq h} \subset \mathbb{R}$ with $x_{0}=0$ we have

$$
\sum_{i=1}^{h-1}\left|x_{i}\left(x_{i+1}-x_{i}\right)\right| \leq \frac{h-1}{2} \sum_{i=0}^{h-1}\left|x_{i+1}-x_{i}\right|^{2},
$$

with equality when $x_{i}=$ ci.

In this paper we prove an Opial inequality that contains both Theorem 1.1 and Theorem 1.2 as special cases. This result is proved in Section 3 and reads as follows.

Theorem 1.3 (Dynamic Opial Inequality). Let $\mathbb{T}$ be a time scale with $0, h \in \mathbb{T}$. For delta differentiable $x:[0, h] \cap \mathbb{T} \rightarrow \mathbb{R}$ with $x(0)=0$ we have

$$
\int_{0}^{h}\left|\left(x+x^{\sigma}\right) x^{\Delta}\right|(t) \Delta t \leq h \int_{0}^{h}\left|x^{\Delta}\right|^{2}(t) \Delta t,
$$

with equality when $x(t)=$ ct.

To see how Theorems 1.1 and 1.2 are special cases of Theorem 1.3, it is at this point sufficient to know that

- $\mathbb{T}=\mathbb{R}$ corresponds to the continuous case, and $x^{\sigma}=x, x^{\Delta}=\dot{x}$, $\int_{0}^{h} f(t) \Delta t=\int_{0}^{h} f(t) \mathrm{d} t$ in this case;

- $\mathbb{T}=\mathbb{Z}$ corresponds to the discrete case, and $x^{\sigma}(t)=x(t+1), x^{\Delta}=$ $x^{\sigma}-x, \int_{0}^{h} f(t) \Delta t=\sum_{t=0}^{h-1} f(t)$ in this case.

A general time scale, however, is an arbitrary closed subset of the reals. Hence our Theorem 1.3 covers many more cases than just the continuous and the discrete ones; see e.g. the examples at the end of the next section, Section 2 , where we give a short introduction to the calculus on time scales. Next, in Section 3, we prove the above Theorem 1.3 and offer an application of this inequality. We also present an Opial inequality that applies to functions $x$ with $x(0)=x(h)=0$ and gives a better bound than the one in Theorem 1.3. Finally, Section 4 contains a number of generalizations of Theorem 1.3, most of which are contained in $[2]$ for the special cases $\mathbb{T}=\mathbb{R}$ and/or $\mathbb{T}=\mathbb{Z}$.

2. The time scales calculus. In this section we briefly introduce the time scales calculus. For proofs and further explanations and results we refer to the papers by Hilger [4, 8, 9], to the book by Kaymakçalan, Lakshmikantham, and Sivasundaram [10], and to the more recent papers $[1,3,5,6,7]$. A time scale $\mathbb{T}$ is a nonempty closed subset of $\mathbb{R}$, and the (forward) jump operator $\sigma: \mathbb{T} \rightarrow \mathbb{T}$ is defined by

$$
\sigma(t)=\inf \{s \in \mathbb{T}: s>t\}
$$


(supplemented by $\inf \emptyset=\sup \mathbb{T}$ ), while the graininess $\mu: \mathbb{T} \rightarrow \mathbb{R}_{+}$is given by

$$
\mu(t)=\sigma(t)-t .
$$

For a function $f: \mathbb{T} \rightarrow \mathbb{R}$ we define the derivative $f^{\Delta}$ as follows: Let $t \in \mathbb{T}$. If there exists a number $\alpha \in \mathbb{R}$ such that for all $\varepsilon>0$ there exists a neighborhood $U$ of $t$ with

$$
|f(\sigma(t))-f(s)-\alpha(\sigma(t)-s)| \leq \varepsilon|\sigma(t)-s| \quad \text { for all } s \in U,
$$

then $f$ is said to be (delta) differentiable at $t$, and we call $\alpha$ the derivative of $f$ at $t$ and denote it by $f^{\Delta}(t)$. Moreover, we write $f^{\sigma}=f \circ \sigma$. The following formulas are useful:

- $f^{\sigma}=f+\mu f^{\Delta}$

- $(f g)^{\Delta}=f^{\Delta} g+f^{\sigma} g^{\Delta}$ ("Product Rule");

- $(f / g)^{\Delta}=\left(f^{\Delta} g-f g^{\Delta}\right) /\left(g g^{\sigma}\right)$ ("Quotient Rule").

A function $F$ with $F^{\Delta}=f$ is called an antiderivative of $f$, and then we define

$$
\int_{a}^{b} f(t) \Delta t=F(b)-F(a)
$$

where $a, b \in \mathbb{T}$. The following existence theorem [8] holds:

- Rd-continuous functions possess antiderivatives.

Here, a function is called $r d$-continuous if it is continuous at right-dense points (i.e., points $t$ where $\sigma(t)=t$ ), and if the left-sided limits exist at leftdense points (i.e., points $t$ where $\varrho(t)=t$, with $\varrho$ being the backward jump operator defined in a natural way similar to the forward jump operator $\sigma$ ). We have (see e.g. [4, Theorem 7])

$$
f(t) \geq 0, a \leq t<b, \quad \text { implies } \quad \int_{a}^{b} f(t) \Delta t \geq 0 .
$$

Throughout this paper we assume that $0 \in \mathbb{T}$ and let $h>0$ with $h \in \mathbb{T}$. Hence, if $\mathbb{T}=\mathbb{R}$, then $\int_{0}^{h} f(t) \Delta t=\int_{0}^{h} f(t) \mathrm{d} t$, and if $\mathbb{T}=\mathbb{Z}$, then $\int_{0}^{h} f(t) \Delta t=$ $\sum_{t=0}^{h-1} f(t)$.

Other examples of time scales (to which our inequalities apply as well) are e.g.

$$
\begin{gathered}
h \mathbb{Z}=\{h k: k \in \mathbb{Z}\} \quad \text { for some } h>0, \\
q^{\mathbb{Z}}=\left\{q^{k}: k \in \mathbb{Z}\right\} \cup\{0\} \quad \text { for some } q>1
\end{gathered}
$$

(which produces so-called $q$-difference equations),

$$
\mathbb{N}^{2}=\left\{k^{2}: k \in \mathbb{N}\right\}, \quad\left\{\sum_{k=1}^{n} \frac{1}{k}: n \in \mathbb{N}\right\}, \quad \bigcup_{k \in \mathbb{Z}}[2 k, 2 k+1],
$$

or the Cantor set. 
We conclude this section with two simple consequences of the product rule as well as Hölder's inequality for time scales. Those results will be needed in Sections 3 and 4. First,

$$
\left(f^{2}\right)^{\Delta}=(f \cdot f)^{\Delta}=f^{\Delta} f+f^{\sigma} f^{\Delta}=\left(f+f^{\sigma}\right) f^{\Delta},
$$

and by induction,

$$
\left(f^{l+1}\right)^{\Delta}=\left\{\sum_{k=0}^{l} f^{k}\left(f^{\sigma}\right)^{l-k}\right\} f^{\Delta}, \quad l \in \mathbb{N} .
$$

Finally, Hölder's inequality for time scales (see [6, Lemma 2.2(iv)]) reads

$$
\int_{0}^{h}|f(t) g(t)| \Delta t \leq\left\{\int_{0}^{h}|f(t)|^{p} \Delta t\right\}^{1 / p}\left\{\int_{0}^{h}|g(t)|^{q} \Delta t\right\}^{1 / q},
$$

where $p>1$ and $q=p /(p-1)$.

3. Opial's inequality and an application. We now prove the simplest version of Opial's inequality on time scales as presented in Theorem 1.3 and then proceed to illustrate the applications of this inequality by means of an example.

Proof of Theorem 1.3. Consider

$$
y(t)=\int_{0}^{t}\left|x^{\Delta}(s)\right| \Delta s
$$

Then $y^{\Delta}=\left|x^{\Delta}\right|$ and $|x| \leq y$ so that

$$
\begin{aligned}
\int_{0}^{h}\left|\left(x+x^{\sigma}\right) x^{\Delta}\right|(t) \Delta t & \leq \int_{0}^{h}\left[\left(|x|+\left|x^{\sigma}\right|\right)\left|x^{\Delta}\right|\right](t) \Delta t \leq \int_{0}^{h}\left[\left(y+y^{\sigma}\right)\left|x^{\Delta}\right|\right](t) \Delta t \\
& =\int_{0}^{h}\left[\left(y+y^{\sigma}\right) y^{\Delta}\right](t) \Delta t=\int_{0}^{h}\left(y^{2}\right)^{\Delta}(t) \Delta t \\
& =y^{2}(h)-y^{2}(0)=\left\{\int_{0}^{h}\left|x^{\Delta}(t)\right| \Delta t\right\}^{2} \\
& \leq h \int_{0}^{h}\left|x^{\Delta}\right|^{2}(t) \Delta t
\end{aligned}
$$

where we have used formulas (2.2) and (2.4) for $p=2$.

Now, let $\widetilde{x}(t)=c t$ for some $c \in \mathbb{R}$. Then $\widetilde{x}^{\Delta}(t) \equiv c$, and it is easy to check that

$$
\int_{0}^{h}\left|\left(\widetilde{x}+\widetilde{x}^{\sigma}\right) \widetilde{x}^{\Delta}\right|(t) \Delta t=h \int_{0}^{h}\left|\widetilde{x}^{\Delta}\right|^{2}(t) \Delta t .
$$


We now give an application of Theorem 1.3.

EXAMPLE 3.1. Let $y$ be a solution of the initial value problem

$$
y^{\Delta}=1-t+y^{2} / t, \quad 0<t \leq 1, \quad y(0)=0 .
$$

Then $y \leq \widetilde{y}$ on $[0,1] \cap \mathbb{T}$, where $\widetilde{y}(t)=t$ solves $(3.1)$.

Proof. Clearly $\widetilde{y}$ as defined above solves (3.1). We let $y$ be a solution of (3.1) and define

$$
R(t)=1-t+\int_{0}^{t}\left|y^{\Delta}\right|^{2}(s) \Delta s .
$$

Let $t \in(0,1] \cap \mathbb{T}$. Then

$$
\begin{aligned}
\left|y^{\Delta}(t)\right| & =\left|1-t+\frac{y^{2}(t)}{t}\right| \leq|1-t|+\frac{1}{t}\left|y^{2}(t)\right| \\
& =1-t+\frac{1}{t}\left|\int_{0}^{t}\left(y^{2}\right)^{\Delta}(s) \Delta s\right| \\
& \leq 1-t+\frac{1}{t} \int_{0}^{t}\left|\left(y^{2}\right)^{\Delta}(s)\right| \Delta s \\
& =1-t+\frac{1}{t} \int_{0}^{t}\left|\left(y+y^{\sigma}\right) y^{\Delta}\right|(s) \Delta s \\
& \leq 1-t+\frac{1}{t} t \int_{0}^{t}\left|y^{\Delta}\right|^{2}(s) \Delta s \\
& =R(t), \quad
\end{aligned}
$$

where we haved used our Opial inequality, Theorem 1.3. Hence

$$
R^{\Delta}(t)=-1+\left|y^{\Delta}(t)\right|^{2} \leq R^{2}(t)-1 \quad \text { and } \quad R(0)=1 .
$$

Let $w$ be the unique solution of

$$
w^{\Delta}=(1+R(t)) w, \quad w(0)=1 .
$$

Note that this $w$ exists since $1+\mu(R+1)>0$ (see e.g. [4, Theorem 8]) because $R>\left|y^{\Delta}\right| \geq 0$; actually $w(t)>0$ for all $t \in[0,1] \cap \mathbb{T}$. Hence, because of $(R-1)^{\Delta}=R^{\Delta} \leq R^{2}-1$, we have

$$
\begin{aligned}
0 & \geq \frac{(R-1)^{\Delta} w-\left(R^{2}-1\right) w}{w w^{\sigma}}=\frac{(R-1)^{\Delta} w-(R-1) w^{\Delta}}{w w^{\sigma}} \\
& =\left(\frac{R-1}{w}\right)^{\Delta}
\end{aligned}
$$

(we used the quotient rule from Section 2) so that 


$$
\left(\frac{R-1}{w}\right)(t)=\left(\frac{R-1}{w}\right)(0)+\int_{0}^{t}\left(\frac{R-1}{w}\right)^{\Delta}(s) \Delta s \leq 0
$$

and hence $R(t) \leq 1$. Therefore

$$
y^{\Delta}(t) \leq\left|y^{\Delta}(t)\right| \leq R(t) \leq 1
$$

and $y(t)=\int_{0}^{t} y^{\Delta}(s) \Delta s \leq \int_{0}^{t} \Delta s=t$.

We next offer a generalization of Theorem 1.3 where $x(0)$ does not need to be 0 . This result is not found in the book [2] (neither a continuous nor a discrete version of it), but both a weaker version of Theorem 1.3 (with $x(0)=0$ ) and the subsequent Theorem 3.2 (with $x(0)=x(h)=0$ ) are corollaries of Theorem 3.1, and continuous and discrete versions of Theorem 3.2 can be found in [2, Theorem 1.3.1 and Theorem 5.2.1, "Lasota's inequality"].

Theorem 3.1. Let $x:[0, h] \cap \mathbb{T} \rightarrow \mathbb{R}$ be differentiable. Then

where

$$
\int_{0}^{h}\left|\left(x+x^{\sigma}\right) x^{\Delta}\right|(t) \Delta t \leq \alpha \int_{0}^{h}\left|x^{\Delta}(t)\right|^{2} \Delta t+2 \beta \int_{0}^{h}\left|x^{\Delta}(t)\right| \Delta t,
$$

$$
\alpha \in \mathbb{T} \quad \text { with } \quad \operatorname{dist}(h / 2, \alpha)=\operatorname{dist}(h / 2, \mathbb{T})
$$

and $\beta=\max \{|x(0)|,|x(h)|\}$.

Proof. We consider

$$
y(t)=\int_{0}^{t}\left|x^{\Delta}(s)\right| \Delta s \quad \text { and } \quad z(t)=\int_{t}^{h}\left|x^{\Delta}(s)\right| \Delta s .
$$

Then $y^{\Delta}=\left|x^{\Delta}\right|, z^{\Delta}=-\left|x^{\Delta}\right|$,

$$
\begin{aligned}
|x(t)| & \leq|x(t)-x(0)|+|x(0)|=\left|\int_{0}^{t} x^{\Delta}(s) \Delta s\right|+|x(0)| \\
& \leq \int_{0}^{t}\left|x^{\Delta}(s)\right| \Delta s+|x(0)|=y(t)+|x(0)|,
\end{aligned}
$$

and similarly $|x(t)| \leq z(t)+|x(h)|$. Let $u \in[0, h] \cap \mathbb{T}$. Then

$$
\begin{aligned}
\int_{0}^{u}\left|\left(x+x^{\sigma}\right) x^{\Delta}\right|(t) \Delta t & \leq \int_{0}^{u}\left[y(t)+|x(0)|+y^{\sigma}(t)+|x(0)|\right] y^{\Delta}(t) \Delta t \\
& =\int_{0}^{u}\left[\left(y+y^{\sigma}\right) y^{\Delta}\right](t) \Delta t+2|x(0)| \int_{0}^{u} y^{\Delta}(t) \Delta t \\
& =y^{2}(u)+2|x(0)| y(u) \\
& \leq u \int_{0}^{u}\left|x^{\Delta}(t)\right|^{2} \Delta t+2|x(0)| \int_{0}^{u}\left|x^{\Delta}(t)\right| \Delta t,
\end{aligned}
$$


where we have used again (2.2) and (2.4) for $p=2$. Similarly, one shows

$$
\begin{aligned}
\int_{u}^{h}\left|\left(x+x^{\sigma}\right) x^{\Delta}\right|(t) \Delta t & \leq z^{2}(u)+2|x(h)| z(u) \\
& \leq(h-u) \int_{u}^{h}\left|x^{\Delta}(t)\right|^{2} \Delta t+2|x(h)| \int_{u}^{h}\left|x^{\Delta}(t)\right| \Delta t .
\end{aligned}
$$

By putting $\nu(u)=\max \{u, h-u\}$ and adding the above two inequalities, we find

$$
\int_{0}^{h}\left|\left(x+x^{\sigma}\right) x^{\Delta}\right|(t) \Delta t \leq \nu(u) \int_{0}^{h}\left|x^{\Delta}(t)\right|^{2} \Delta t+2 \beta \int_{0}^{h}\left|x^{\Delta}(t)\right| \Delta t .
$$

This is true for any $u \in[0, h] \cap \mathbb{T}$, so it is also true if $\nu(u)$ is replaced by $\min _{u \in[0, h] \cap \mathbb{T}} \nu(u)$. However, this last quantity is easily seen to be equal to $\alpha$.

Theorem 3.2. Suppose that $x:[0, h] \cap \mathbb{T} \rightarrow \mathbb{R}$ is differentiable with $x(0)=x(h)=0$. Then

$$
\int_{0}^{h}\left|\left(x+x^{\sigma}\right) x^{\Delta}\right|(t) \Delta t \leq \alpha \int_{0}^{h}\left|x^{\Delta}(t)\right|^{2} \Delta t
$$

where $\alpha$ is given in (3.2).

Proof. This follows easily from Theorem 3.1 since in this case $\beta=0$.

4. Extensions. Here we wish to offer some generalizations of the inequalities presented above. The continuous and/or discrete versions of those inequalities may be found in [2]. We have not included all of those results in this paper, but most of them may be proved using similar techniques.

THEOREM 4.1 (see [2, Theorem 2.5.1]). Let $p, q$ be positive and continuous on $[0, h], \int_{0}^{h} \Delta t / p(t)<\infty$, and $q$ nonincreasing. For differentiable $x:[0, h] \cap \mathbb{T} \rightarrow \mathbb{R}$ with $x(0)=0$ we have

$$
\int_{0}^{h}\left[q^{\sigma}\left|\left(x+x^{\sigma}\right) x^{\Delta}\right|\right](t) \Delta t \leq\left\{\int_{0}^{h} \frac{\Delta t}{p(t)}\right\}\left\{\int_{0}^{h} p(t) q(t)\left|x^{\Delta}(t)\right|^{2} \Delta t\right\} .
$$

Proof. We consider

$$
y(t)=\int_{0}^{t} \sqrt{q^{\sigma}(s)}\left|x^{\Delta}(s)\right| \Delta s .
$$

Then $y^{\Delta}=\sqrt{q^{\sigma}}\left|x^{\Delta}\right|$ and (note that $0 \leq s<t$ implies $\sigma(s) \leq t$ and hence $q(\sigma(s)) \geq q(t) ;$ apply $(2.1))$

$$
|x(t)| \leq \int_{0}^{t}\left|x^{\Delta}(s)\right| \Delta s \leq \int_{0}^{t} \sqrt{\frac{q^{\sigma}(s)}{q(t)}}\left|x^{\Delta}(s)\right| \Delta s=\frac{y(t)}{\sqrt{q(t)}}
$$


so that (note that $t \leq \sigma(t)$ implies $q(t) \geq q(\sigma(t))$ )

$$
\begin{aligned}
\int_{0}^{h}\left[q^{\sigma}\left|\left(x+x^{\sigma}\right) x^{\Delta}\right|\right](t) \Delta t & \leq \int_{0}^{h} q^{\sigma}(t)\left(\frac{y(t)}{\sqrt{q(t)}}+\frac{y^{\sigma}(t)}{\sqrt{q^{\sigma}(t)}}\right) \frac{y^{\Delta}(t)}{\sqrt{q^{\sigma}(t)}} \Delta t \\
& \leq \int_{0}^{h}\left(y(t)+y^{\sigma}(t)\right) y^{\Delta}(t) \Delta t \\
& =y^{2}(h) \\
& =\left\{\int_{0}^{h} \frac{1}{\sqrt{p(s)}} \sqrt{p(s) q^{\sigma}(s)}\left|x^{\Delta}(s)\right| \Delta s\right\}^{2} \\
& \leq\left\{\int_{0}^{h} \frac{\Delta s}{p(s)}\right\}\left\{\int_{0}^{h} p(s) q^{\sigma}(s)\left|x^{\Delta}(s)\right|^{2} \Delta s\right\} .
\end{aligned}
$$

Again we have used (2.2) and (2.4) for $p=2$.

The following result involves higher order derivatives. As usual, we write $f^{\Delta^{n}}$ for the $n$th (delta) derivative of $f$.

Theorem 4.2 (see $[2$, Chapter 3$])$. Suppose $l, n \in \mathbb{N}$. For $n$-times differentiable $x:[0, h] \cap \mathbb{T} \rightarrow \mathbb{R}$ with $x(0)=x^{\Delta}(0)=\ldots=x^{\Delta^{n-1}}(0)=0$ we have

$$
\int_{0}^{h}\left|\left\{\sum_{k=0}^{l} x^{k}\left(x^{\sigma}\right)^{l-k}\right\} x^{\Delta^{n}}\right|(t) \Delta t \leq h^{l n} \int_{0}^{h}\left|x^{\Delta^{n}}(t)\right|^{l+1} \Delta t .
$$

Proof. We consider

$$
y(t)=\int_{0}^{t} \int_{0}^{\tau_{n-1}} \ldots \int_{0}^{\tau_{2}}\left\{\int_{0}^{\tau_{1}}\left|x^{\Delta^{n}}(s)\right| \Delta s\right\} \Delta \tau_{1} \Delta \tau_{2} \ldots \Delta \tau_{n-1} .
$$

Hence we have

$$
\begin{aligned}
y^{\Delta}(t) & =\int_{0}^{t} \int_{0}^{t} \ldots \int_{0}^{\tau_{n}}\left\{\int_{0}^{\tau_{1}}\left|x^{\Delta^{n}}(s)\right| \Delta s\right\} \Delta \tau_{1} \Delta \tau_{2} \ldots \Delta \tau_{n-2}, \ldots, \\
y^{\Delta^{n-1}}(t) & =\int_{0}^{t}\left|x^{\Delta^{n}}(s)\right| \Delta s, \quad y^{\Delta^{n}}(t)=\left|x^{\Delta^{n}}(t)\right|,
\end{aligned}
$$

and for $0 \leq t \leq h$,

$$
\begin{aligned}
|x(t)| & \leq \int_{0}^{t}\left|x^{\Delta}\left(t_{1}\right)\right| \Delta t_{1} \leq \int_{0}^{t} \int_{0}^{t_{1}}\left|x^{\Delta \Delta}\left(t_{2}\right)\right| \Delta t_{2} \Delta t_{1} \leq \ldots \leq y(t) \\
& =\int_{0}^{t} y^{\Delta}(s) \Delta s \leq \int_{0}^{t} y^{\Delta}(t) \Delta s \leq \int_{0}^{h} y^{\Delta}(t) \Delta s=h y^{\Delta}(t) \\
& \leq h^{2} y^{\Delta \Delta}(t) \leq \ldots \leq h^{n-1} y^{\Delta^{n-1}}(t)=h^{n-1} f(t),
\end{aligned}
$$


where we put $f=y^{\Delta^{n-1}}$. Therefore

$$
\begin{aligned}
& \int_{0}^{h}\left|\left\{\sum_{k=0}^{l} x^{k}\left(x^{\sigma}\right)^{l-k}\right\} x^{\Delta^{n}}\right|(t) \Delta t \leq \int_{0}^{h}\left\{\sum_{k=0}^{l}|x|^{k}\left|x^{\sigma}\right|^{l-k}\left|x^{\Delta^{n}}\right|\right\}(t) \Delta t \\
& \leq \int_{0}^{h}\left\{\sum_{k=0}^{l}\left(h^{n-1} f\right)^{k}\left(h^{n-1} f^{\sigma}\right)^{l-k}\left|x^{\Delta^{n}}\right|\right\}(t) \Delta t \\
&=h^{(n-1) l} \int_{0}^{h}\left\{\sum_{k=0}^{l} f^{k}\left(f^{\sigma}\right)^{l-k} f^{\Delta}\right\}(t) \Delta t=h^{(n-1) l} \int_{0}^{h}\left(f^{l+1}\right)^{\Delta}(t) \Delta t \\
&=h^{(n-1) l} f^{l+1}(h)=h^{(n-1) l}\left\{\int_{0}^{h}\left|x^{\Delta^{n}}(t)\right| \Delta t\right\}^{l+1} \\
& \leq h^{(n-1) l} h^{l} \int_{0}^{h}\left|x^{\Delta^{n}}(t)\right|^{l+1} \Delta t=h^{n l} \int_{0}^{h}\left|x^{\Delta^{n}}(t)\right|^{l+1} \Delta t,
\end{aligned}
$$

where we have used (2.1), (2.3), and (2.4) with $p=(l+1) / l$ and $q=l+1$.

The following two results are easy corollaries of the above Theorem 4.2.

Corollary 4.1 (see [2, Theorem 3.2.1]). Suppose $n \in \mathbb{N}$. For $n$-times differentiable $x:[0, h] \cap \mathbb{T} \rightarrow \mathbb{R}$ with $x(0)=x^{\Delta}(0)=\ldots=x^{\Delta^{n-1}}(0)=0$ we have

$$
\int_{0}^{h}\left|\left(x+x^{\sigma}\right) x^{\Delta^{n}}\right|(t) \Delta t \leq h^{n} \int_{0}^{h}\left|x^{\Delta^{n}}(t)\right|^{2} \Delta t .
$$

Proof. This is Theorem 4.2 with $l=1$.

Corollary 4.2 (see [2, Theorem 2.3.1]). Suppose $l \in \mathbb{N}$. For differentiable $x:[0, h] \cap \mathbb{T} \rightarrow \mathbb{R}$ with $x(0)=0$ we have

$$
\int_{0}^{h}\left|\left\{\sum_{k=0}^{l} x^{k}\left(x^{\sigma}\right)^{l-k}\right\} x^{\Delta}\right|(t) \Delta t \leq h^{l} \int_{0}^{h}\left|x^{\Delta}(t)\right|^{l+1} \Delta t .
$$

Proof. This is Theorem 4.2 with $n=1$.

\section{References}

[1] R. P. Agarwal and M. Bohner, Basic calculus on time scales and some of its applications, Results Math. 35 (1999), 3-22.

[2] R. P. Agarwal and P. Y. H. Pang, Opial Inequalities with Applications in Differential and Difference Equations, Kluwer, Dordrecht, 1995.

[3] C. D. Ahlbrandt, M. Bohner, and J. Ridenhour, Hamiltonian systems on time scales, J. Math. Anal. Appl. 250 (2000), 561-578. 
[4] B. Aulbach and S. Hilger, Linear dynamic processes with inhomogeneous time scale, in: Nonlinear Dynamics and Quantum Dynamical Systems (Gaussig, 1990), Math. Res. 59, Akademie-Verlag, Berlin, 1990, 9-20.

[5] M. Bohner, S. Clark, and J. Ridenhour, Lyapunov inequalities on time scales, J. Inequal. Appl. (2000), to appear.

[6] M. Bohner and D. A. Lutz, Asymptotic behavior of dynamic equations on time scales, J. Differ. Equations Appl. 7 (2001), 21-50.

[7] O. Došlý and R. Hilscher, Disconjugacy, transformations and quadratic functionals for symplectic dynamic systems on time scales, ibid., to appear.

[8] S. Hilger, Analysis on measure chains - a unified approach to continuous and discrete calculus, Results Math. 18 (1990), 18-56.

[9] -, Special functions, Laplace and Fourier transform on measure chains, Dynam. Systems Appl. 8 (1999), 471-488 (Special Issue on "Discrete and Continuous Hamiltonian Systems", edited by R. P. Agarwal and M. Bohner).

[10] B. Kaymakçalan, V. Lakshmikantham, and S. Sivasundaram, Dynamic Systems on Measure Chains, Math. Appl. 370, Kluwer, Dordrecht, 1996.

[11] A. Lasota, A discrete boundary value problem, Ann. Polon. Math. 20 (1968), 183190.

[12] Z. Opial, Sur une inégalité, ibid. 8 (1960), 29-32.

Department of Mathematics and Statistics

University of Missouri-Rolla

Rolla, MO 65409-0020, U.S.A.

Department of Mathematics

E-mail: bohner@umr.edu Middle East Technical University 06531 Ankara, Turkey E-mail: billur@metu.edu.tr 